\title{
Measuring the Spins of Stellar Black Holes: A Progress Report
}

\section{Citation}

McClintock, J. E., R. Narayan, L. Gou, J. Liu, R.F. Penna, J.F. Steiner, A. Comastri, L. Angelini, and M. Cappi. 2010. Measuring the spins of stellar black holes: A progress report. AIP Conference Proceedings 1248 (101): 101-106.

\section{Published Version}

10.1063/1.3475156

\section{Permanent link}

http://nrs.harvard.edu/urn-3:HUL.InstRepos:27231581

\section{Terms of Use}

This article was downloaded from Harvard University's DASH repository, and is made available under the terms and conditions applicable to Open Access Policy Articles, as set forth at http:// nrs.harvard.edu/urn-3:HUL.InstRepos:dash.current.terms-of-use\#OAP

\section{Share Your Story}

The Harvard community has made this article openly available.

Please share how this access benefits you. Submit a story.

\section{Accessibility}




\title{
Measuring the Spins of Stellar Black Holes: A Progress Report
}

\author{
J. E. McClintock, R. Narayan, L. Gou, J. Liu, R. F. Penna and J. F. Steiner \\ Harvard-Smithsonian Center for Astrophysics, 60 Garden St., Cambridge, MA 02138 USA
}

\begin{abstract}
We use the Novikov-Thorne thin disk model to fit the thermal continuum X-ray spectra of black hole X-ray binaries, and thereby extract the dimensionless spin parameter $a_{*}=a / M$ of the black hole as a parameter of the fit. We summarize the results obtained to date for six systems and describe work in progress on additional systems. We also describe recent methodological advances, our current efforts to make our analysis software fully available to others, and our theoretical efforts to validate the Novikov-Thorne model.
\end{abstract}

Keywords: X-ray sources; X-ray binaries; Accretion and accretion disks

PACS: 98.70Qy; 97,80.Jp; 97.10.Gz

\section{INTRODUCTION}

We now know of about 40 stellar-mass black holes (BHs) in X-ray binaries in the Milky Way and neighboring galaxies with masses ranging from $\sim 5-20 M_{\odot}[1]$. Astrophysical BHs are completely described by the two numbers that specify their mass and spin. $\mathrm{BH}$ spin is commonly expressed in terms of the dimensionless quantity $a_{*} \equiv c J / G M^{2}$ with $\left|a_{*}\right| \leq 1$, where $M$ and $J$ are respectively the $\mathrm{BH}$ mass and angular momentum. Currently, there are two techniques that are delivering measurements of spin, namely fitting the thermal X-ray continuum [2-6] and modeling the profile of the Fe K line. Our group is engaged in using the continuum-fitting $(\mathrm{CF})$ method, which is the focus of this paper. (For a discussion of the Fe K method see [7]).

Knowledge of BH spin is crucial for answering many key questions. For example: Are relativistic jets powered by spin? What role does spin play in producing a gamma-ray burst? What constraints can be placed on models of supernovae, $\mathrm{BH}$ formation, and $\mathrm{BH}$ binary evolution? What distribution of BH spins should LIGO waveform modelers be considering? For supermassive BHs, is the distribution of spins of the merging partners consistent with hierarchical models for their growth?

In the following two sections, we describe the CF method of determining spin and present the results we have obtained to date, while describing our current work on four additional sources. The next section describes recent methodological advances, including our efforts to make all of our fitting software publicly available. The penultimate section describes our work aimed at validating the theoretical underpinning of our work - the Novikov-Thorne disk model - via GRMHD simulations. We conclude with a number of questions that motivate us. 


\section{THE METHOD: FITTING THE X-RAY CONTINUUM SPECTRUM}

Here we present the bare elements of the method; for a fuller explanation, see Sec. 2 in [9]. The foundation of the $\mathrm{CF}$ method is the definite prediction of relativity theory that there exists an innermost stable circular orbit (ISCO) for a test particle orbiting a $\mathrm{BH}$. We identify the radius of the ISCO, $R_{\mathrm{ISCO}}$, with the inner edge of the accretion disk. Thus, the gas spirals in (through the action of viscosity) via a series of nearly circular orbits until it reaches the ISCO, at which point it plunges into the $\mathrm{BH}$. In our method, we estimate the radius of the inner edge of the disk by fitting the X-ray continuum spectrum and identify this radius with $R_{\mathrm{ISCO}}$. Since the dimensionless ratio $\xi \equiv R_{\mathrm{ISCO}} /\left(G M / c^{2}\right)$ is solely a monotonic function of the BH spin parameter $a_{*}$, knowing its value allows one immediately to infer the $\mathrm{BH}$ spin parameter $a_{*}$. The variations in $R_{\text {ISCO }}$ are large, ranging from $6 G M / c^{2}$ to $G M / c^{2}$ as $a_{*}$ increases from 0 to unity.

Thousands of observations of $\mathrm{BH}$ binaries in the thermal state, which have been made during the past 25 years, suggest that fitting the X-ray continuum is a promising approach to measuring BH spin. Specifically, these observations have provided abundant evidence for a constant inner disk radius in the thermal state. For discussions of this empirical foundation of the method see $[6,10]$.

The idealized thin disk model of Novikov \& Thorne [8] describes an axisymmetric radiatively-efficient accretion flow in which, for a given $\mathrm{BH}$ mass $M$, mass accretion rate $\dot{M}$ and BH spin parameter $a_{*}$, we can calculate precisely the total luminosity of the disk, $L_{\text {disk }}=\eta \dot{M} c^{2}$, where the radiative efficiency factor $\eta$ is a function only of $a_{*}$. Moreover, the accreting gas is optically thick, and the emission is thermal and blackbody-like, making it straightforward to compute the spectrum of the emission and other properties of the disk, such as its luminosity profile $L(R)$. Most importantly, as discussed above, the inner edge of the disk is located at the ISCO of the BH space-time. By analyzing the spectrum of the disk radiation and combining it with knowledge of the distance $D$, the inclination angle $i$ and the mass $M$ of the $\mathrm{BH}$, we can obtain $a_{*}$. This is the principle behind our method of estimating BH spin, which was first described by Zhang et al. [11].

For the $\mathrm{CF}$ method to succeed it is essential to have accurate measurements of the $\mathrm{BH}$ mass $M$, inclination of the accretion disk $i$, and distance $D$ as inputs to the continuumfitting process $[2,3]$. This dynamical work is not discussed here, although about half of our total observational effort is directed toward securing these dynamical data (e.g., see $[12,13])$.

\section{RESULTS TO DATE AND WORK IN PROGRESS}

In the top portion of Table 1, we summarize the half-dozen measurements of spin obtained using the $\mathrm{CF}$ method during the past four years. The values of spin range widely: LMC X-3 is a near Schwarzschild hole and GRS $1915+105$ is a near-extreme Kerr hole, while four sources have intermediate values of spin in the range $a_{*} \sim 0.7-$ 0.9. The values of $\mathrm{BH}$ mass used in deriving the spin estimates are given in the table. For the details on this published work we refer the reader to the papers cited, and we now turn to comment on the four sources listed at the bottom of the table. 
TABLE 1. Spin Measurements of Stellar Black Holes: Published and in the Works

\begin{tabular}{rrrl}
\hline BH Binary System & \multicolumn{1}{c}{$M / M_{\odot}$} & \multicolumn{1}{c}{$a_{*}$} & References \\
\hline 4U 1543-47 & $9.4 \pm 1.0$ & $0.75-0.85$ & {$[2]$} \\
GRO J1655-40 & $6.30 \pm 0.27$ & $0.65-0.75$ & {$[2]$} \\
GRS 1915+105 & $14 \pm 4.4$ & $0.98-1$ & {$[3]$} \\
LMC X-3 & $7(5-11)$ & $<0.26$ & {$[4]$} \\
M33 X-7 & $15.65 \pm 1.45$ & $0.77 \pm 0.05$ & {$[5,12]$} \\
LMC X-1 & $10.91 \pm 1.41$ & $0.92_{-0.07}^{+0.05}$ & {$[6,13]$} \\
XTE J1550-564 & TBD & TBD & Steiner et al.; Orosz et al. \\
A0620-00 & TBD & TBD & Gou et al.; [14] \\
Cygnus X-1 & TBD & TBD & Gou et al.; Orosz et al.; Reid et al. \\
LMC X-3 & TBD & TBD & Steiner et al.; Orosz et al. \\
\hline
\end{tabular}

XTE J1550-564: Extensive photometric and spectroscopic data have been collected, and a paper on a new dynamical model of the system is in preparation, which will supersede our earlier model [15]. Meanwhile, a preliminary spin analysis of 136 RXTE spectra has already been published [16], and we are eagerly working toward a definitive measurement of the spin of this pc-scale ballistic jet source.

A0620-00: This system, which brightened to an unprecedented 50 Crab in 1975, is the prototype of the nine short-period $\mathrm{BH}$ binaries $\left(P_{\text {orb }}<12 \mathrm{hr}\right)$ [1]. Using a new determination of the $\mathrm{BH}$ mass, inclination and distance, which is based on an exhaustive study of the available photometric data [14], we are in the process of determining the spin using HEASARC archival data obtained in 1975 by the OSO- 8 satellite.

Cygnus X-1: Within several months, we expect to have an accurate VLBA parallax measurement of the distance to this source (Reid et al.), which will be followed by dynamical modeling and a determination of the $\mathrm{BH}$ mass and inclination. Meanwhile, we are working to determine the spin using ASCA GIS data and RXTE PCA data, which were obtained simultaneously [17].

$L M C X$-3: This source appears twice in Table 1. The spin constraint given in line 4 is not firm because it is based on an early and uncertain estimate of the BH mass [4]. We have recently obtained extensive and very high quality spectroscopic and photometric data and are in the process of revisiting the dynamical model. An extensive spin analysis of the X-ray data has already been completed [18].

\section{RECENT ADVANCES IN METHODOLOGY}

As we now describe, using new techniques we are able to both successfully apply the CF method to a larger body of data and to derive uncertainties in the spin parameter that include all of the observational uncertainties. In this section we also describe our tentative plans for improving our relativistic code and making it fully accessible via XSPEC.

Beyond the Thermal Dominant State: All of our prior work on measuring spins has relied on the use of weakly Comptonized spectra obtained in the thermal dominant (TD) state. Now, however, using our recently-developed empirical model of Comptonization 
SIMPL [19], which is available in XSPEC, we are able to obtain values of spin that are consistent with those obtained in the TD state. We have demonstrated this capability by analyzing many RXTE spectra of two BH transients, H1743-322 and XTE J1550-564, and showing that the radius of the inner edge of their accretion disks remains constant to within a few percent as the strength of the Comptonized component increases by an order of magnitude, i.e., as the fraction of the thermal seed photons that are scattered approaches $25 \%$ [16]. This development allows us to apply the CF method to a much wider body of data than previously thought possible, and potentially to sources that have never been observed to enter the TD state.

Monte-Carlo Error Analysis: In early work [2,3], we made only quite approximate estimates of the error in the spin parameter. In contrast, the error analysis in our most recent papers on M33 X-7 and LMC X-1 is much more sophisticated [5,6]. Therein, we determine the error in $a_{*}$ due to the combined uncertainties in $M, i$, and $D$ via Monte Carlo simulations assuming that the uncertainties in these parameters are normally and independently distributed. Most recently, for LMC X-1 we also performed a combined error analysis that considers both of our fiducial values of the viscosity parameter (see Fig. 8 in [6]); thus the error in this case includes the uncertainty in this key model parameter as well as all sources of observational error. Meanwhile, the largest error in our results arise from uncertainties in the validity of the disk model we employ (see below). Another source of uncertainty is our assumption that the spin of the $\mathrm{BH}$ is aligned with the orbit vector to within a few degrees. This question of relative alignment will be addressed directly through observations using the GEMS mission Xray polarimeter now scheduled for flight in 2014 [20,21].

Improvements in Relativistic Disk Codes and Public Access: Our workhorse accretion disk model is available in XSPEC under the name KERRBB [22]. It includes all relativistic effects and additional features; most importantly it includes self-irradiation of the disk ("returning radiation"). A limitation of KERRBB is that one of its three key fit parameters, namely, the spectral hardening factor $f$, is treated as a constant. Because of this limitation, our work is also based on a second, complementary relativistic disk model called BHSPEC [23,24], which is also implemented in XSPEC. The model BHSPEC does not include the effects of returning radiation, but it does provide state-of-the-art capability for computing the spectral hardening factor $f$. In all of our work since Shafee et al. [2], we have used a hybrid code that combines the functionalities of BHSPEC and KERRBB into a single code we call KERRBB2 (see Sec. 4.2 of [3] for details).

It is quite awkward to analyze data using the hybrid code KERRBB2 because for each fit one must first use BHSPEC to generate a large multi-dimensional table of the spectral hardening factor $f$, which depends on the relevant detector response function, and then read values of $f$ from this table while performing the fit using KERRBB. We are finding that it is quite difficult to implement KERRBB2 for public use within XSPEC. Consequently, we are now developing a new and simpler hybrid code BHSPEC 2 that uses BHSPEC as the primary engine and includes the effects of returning radiation computed using KERRBB. The significant advantage of this approach is that it is independent of detector response and is therefore simpler. Our goal is to make BHSPEC 2 publicly available in XSPEC during the first half of 2010. Our further, three-year goal is to create a new version of BHSPEC that organically includes returning radiation. 


\section{TESTING THE NOVIKOV-THORNE MODEL}

Any measurement of BH spin is only as good as the theoretical model behind it. The $\mathrm{CF}$ method assumes that the radial luminosity profile of the disk $L(r)$ is given by the analytical form derived by Novikov \& Thorne [8]. However, the validity of the NT model, and in particular the zero-torque boundary condition at the ISCO, which it assumes, has been questioned [25,26]. Because any serious error in the NT model will lead to large systematic errors in the derived $\mathrm{BH}$ spin values, we have mounted a major effort to scrutinize the NT model.

We are carrying out GRMHD simulations of thin accretion disks in the Kerr metric and comparing the simulation results with the predictions of the NT model. Our first results for a nonspinning $\mathrm{BH}$ are reported in Shafee et al. [2] where we show that, for a disk with a dimensionless thickness parameter $H / R \sim 0.05$, there is little evidence for significant magnetic coupling across the ISCO. In particular, the angular momentum profile of the simulated flow agrees very closely ( $<2 \%$ difference) with the NT prediction. In ongoing work, we are simulating disks of a variety of thicknesses, $H / R \sim 0.05$, $0.1,0.2,0.3$, around BHs of various spins, $a_{*}=0,0.7,0.9,0.98$. Once again we find that, for all four values of $a_{*}$, the thinnest disk models closely resemble the NT model in terms of their angular momentum profiles, whereas thicker disks show progressively larger deviations. This is an extremely encouraging result for our BH spin program. All our spin estimates to date have been obtained using X-ray spectral data on relatively low-luminosity systems with $L_{\text {disk }} / L_{\text {Edd }} \leq 0.3$. At these luminosities, the disks are estimated to have $H / R \leq 0.1$ [3], suggesting that the spin estimates we have reported so far are robust.

We are also extracting from the GRMHD simulations the luminosity profiles $L(r)$ of the various disk models with a view to comparing these with the predictions of the NT model. The function $L(r)$, which is the most crucial model input for the CF method, is unfortunately less easy to estimate accurately from simulations; it involves taking the difference of two large quantities, whereas the angular momentum profile discussed earlier does not involve such a difference. Nevertheless, our current results are encouraging in the sense that, for thinner disks, our numerically derived profiles of $L(r)$ seem to agree well with the analytical $L(r)$ of the NT model. All our spin estimates have been obtained using the latter profile, so we continue to be confident in the spin estimates listed in Table 1. Meanwhile, we are pushing ahead with additional state-ofthe-art GRMHD simulations of thin and thick disks and hope to have definitive results shortly.

\section{CONCLUSION}

We conclude with a list of questions that motivate us as we work toward our goal of measuring the spins of a dozen or more BHs. What range and distribution of spins will we find? Will GRS 1915+105 stand alone, or will we find other examples of extreme spin? As we continue to refine our models and our measurements of $M, i$ and $D$, will we consistently find values of $a_{*}<1$, or will we be challenged by apparent and unphysical values of the spin parameter that exceed unity? Will all our spin estimates be 
positive, or will we find some BHs with $a_{*}<0$, corresponding to a counter-rotating disk? Will there be large differences in spin between the class of young, persistent systems with their massive secondaries (Cyg X-1, M33 X-7, LMC X-1 and LMC X-3) and the ancient transient systems with their low-mass secondaries? What constraints will these spin results place on $\mathrm{BH}$ formation, evolutionary models of $\mathrm{BH}$ binaries, models of relativistic jets and gamma-ray bursts, etc.? What will be the implications of these spin measurements for the emerging field of gravitational-wave astronomy in the Advanced LIGO era? How will this new knowledge help shape the observing programs of IXO, LISA and other future space missions?

\section{REFERENCES}

1. R. A. Remillard, and J. E. McClintock, ARAA 44, 49-92 (2006).

2. R. Shafee, J. E. McClintock, R. Narayan, S. W. Davis, L. Li and R. A. Remillard, ApJ 636, L113-L116 (2006).

3. J. E. McClintock, R. Shafee, R. Narayan, R. A. Remillard, S. W. Davis and L. Li, ApJ 652, 518-539 (2006).

4. S. W. Davis, C. Done and O. M. Blaes, ApJ 647, 525-538 (2006).

5. J. Liu, J. E. McClintock, R. Narayan, S. W. Davis and J. A. Orosz, ApJ 679, L37-L40 (2008).

6. L. Gou, J. E. McClintock, R. Narayan, et al., ApJ 701, 1076-1090 (2009).

7. J. M. Miller, ARAA 45, 441-479 (2007).

8. I. D. Novikov and K. S. Thorne, "Black Hole Astrophysics," in Black Holes, edited by C. DeWitt and B. DeWitt, Gordan and Breach, New York, 1973, pp. 343-450.

9. R. Narayan, J. E. McClintock and R. Shafee, "Estimating the Spins of Stellar-Mass Black Holes by Fitting Their Continuum Spectra," AIP Conference Proceedings 968, American Institute of Physics, New York, 2008, pp. 265-272.

10. J. E. McClintock, R. Narayan and R. Shafee, "Estimating the Spins of Stellar-Mass Black Holes," to appear in Black Holes, edited by M. Livio and A. Koekemoer, Cambridge Univ. Press, Cambridge (2010; arXiv:0707.4492v1 [astro-ph])

11. S. N. Zhang, W. Cui and W. Chen, ApJ 482, L155-L158 (1997).

12. J. A. Orosz, J. E. McClintock, R. Narayan, et al., Nature 449, 872-875 (2007).

13. J. A. Orosz, D. Steeghs, J. E. McClintock, et al., ApJ 697, 573-591 (2009).

14. A. G. Cantrell, C. D. Bailyn, J. A. Orosz, et al., ApJ, submitted (2009).

15. J. A. Orosz, P. J. Groot, M. van der Klis, et al., ApJ 568, 845-861 (2002).

16. J. F. Steiner, J. E. McClintock, R. A. Remillard, R. Narayan and L. Gou, ApJ 701, L83-L86 (2009).

17. W. Cui, K. Ebisawa, T. Dotani and A. Kubota, ApJ 493, L75-L78 (1998).

18. J. F. Steiner, et al., in preparation.

19. J. F. Steiner, R. Narayan, J. E. McClintock and K. Ebisawa, PASP 121, 1279 (2009).

20. P. Karret, J. Swank, K. Jahoda, T. Kallman, et al., "The Gravity and Extreme Magnetism Small Explorer (GEMS)," to appear in Chandra's First Decade of Discovery," edited by S. Wolk et al. (2009)

21. L.-X. Li, R. Narayan and J. E. McClintock, ApJ 691, 847-865 (2009)

22. L.-X. Li, E. R. Zimmerman, R. Narayan and J. E. McClintock, ApJS 157, 335-370 (2005).

23. S. W. Davis, O. M. Blaes, I. Hubeny and N. J. Turner, ApJ 621, 372-387 (2005).

24. S. W. Davis and I. Hubeny, ApJS 164, 530-535 (2006).

25. J. Krolik, ApJ 515, L73-L76 (1999).

26. C. F. Gammie, ApJ 522, L57-L60 (1999). 